\title{
Subject Index Vol. 8, 1995
}

\section{A431 279}

Acetaminophen 279

Acnevulgaris 319

Active oxygen species 1

Adaptation of penetration profiles 119

Age 246

All-írarø-retinoic acid 167

Anthrone derivatives, novel 1

Anthrones, antipsoriatic 1

Anti-inflammatory properties 211

Antimycotic agent 211

Antipsoriatic agents 41

Arachidonic acid metabolism 196,211

Atopic dermatitis 113

Attenuated total reflectance-Fourier transform infrared spectroscopy 229

Azole derivatives 326

Balding stumptail macaque 221 Barrier function 179 Benzoylperoxide 130 Blood 326

Calcium 309 Carcinogenesis 130 Chemical mediators 113 Chloroquine, low-dose 156

Classification 252 Clofibric acid 203 Contact hypersensitivity 300 Corneocyte cohesion 173

CRABP 167 Croconazole 211, 326 Cyclosporine 149 Cytochrome P450 162 Cytoprotection 279

Dapsone 196 Desquamation 173 Dithranol 1, 119

Eicosanoid inhibition 326 Electrical admittance 237 - capacitance 179

Electrophoresis 167 Erythromycin 319 Essential fatty acids 30 Excised human skin 119

Fatty acid metabolism 30

- $\quad$ - uptake 30

Fibroblasts 139

Flavonoids 279

Free radicals 139

Friction coefficient 246

Glutathione peroxidase 139 Guinea pigs 113

Hamster flank organ 203

HepG2 279

HER-2 215

Histamine 113

Human epidermoid cell line 279

- $\quad$ hepatoblastoma cell line 279

Hydration, skin 237

Hypertrichotic agents 309

IgE-mediated reactions 113 IL-8 215 
Image analysis 252 Inflammation $300 \gamma$-Interferon 41 Interferons 162 Interleukin-1 41,149

Interleukin-6 149 In vitro cytotoxicology 279 In vivo models 292 Ion pair 319 Iontophoresis 229

Irritancy testing, in vitro 49

Keratinocyte(s) 30, 41, 130, 309 Keratolytic effect 173

Laser Doppler flowmetry 229 Lipogenesis 203

5-Lipoxygenase inhibition 1,196 Liver 162

Living skin equivalent 49 Luffariellolide 300

337

Macrophage 300 Manoalide 300 Mast cells 25 Microdialysis 113 Microtopography 252

Minoxidil 221 Moisture, skin 237 Monooxygenases 162 mRNA 167

Multilayer membrane system 11319

Osteoporosis 207 Oxidative stress 139

p53 215

Partition coefficient 319

Penetration 319

- $\quad$ enhancer 221

Phospholipase A2 300

Polymorphonuclear leukocytes

326

- $\quad$-, human 196

Porphyria cutanea tarda 156

Potassium channel openers 309

Proliferation 309

Protein kinase C 130 Psoriasis 25,215

Reconstructed epidermis on

deepidermized dermis 49 Regional variability 246 Retinoicacid 292 Retinoids 292

Salicylic acid 173 Scalaradial 300 Scalp hair growth 221 Selenium 139 SEPATM $^{\text {TM }} 21$ Sex 246

Silymarin 279 Skin 113,162,229

absorption 173

recombinants, human 49

relief 252

thickness 207 Sodium lauryl sulfate 49 Steroids 215

Stratum corneum 179,229 Surface lipid content 246 Swelling test, mouse ear 211

Tape stripping 173

Target organ toxicity 279

Three-electrode system 237

Tiacrilast 25

$\mathrm{TNF}<\mathrm{x} 215$

Transepidermal water loss 179 ,

229 Tumor necrosis factor 149

Ultrasound A scan 207 Ultraviolet A 139 Urea 173

Vitamin A 292

Subject Index Vol. 8,1995 